\title{
Stability and Stabilizability of Switched Linear Systems: A Short Survey of Recent Results
}

\author{
Hai Lin and Panos J. Antsaklis
}

\begin{abstract}
During the last decade, there has been increasing interest in the stability analysis and switching control design for switched linear systems. This paper aims to briefly survey recent results in this field, focusing on stability analysis and switching stabilization problems. First, the stability analysis problem for switched linear systems is reviewed. We focus on the asymptotic stability analysis for switched linear systems under arbitrary switching, and highlight necessary and sufficient conditions for this problem. Secondly, the switching stabilization problem is studied, and a variety of switching stabilization methods found in the literature are outlined. One of the most elusive problems in the switched systems literature has been the switching stabilizability problem, that is under what condition it is possible to stabilize a switched system by properly designing switching control laws. Necessary and sufficient conditions for asymptotic stabilizability of switched linear systems are described.
\end{abstract}

\section{INTRODUCTION}

A switched system is a dynamical system that consists of a finite number of subsystems and a logical rule that orchestrates switching between these subsystems. Mathematically, these subsystems are usually described by a collection of indexed differential or difference equations. In this paper, we will focus on switched systems whose subsystems are continuous-time linear time-invariant (LTI) systems

$$
\dot{x}(t)=A_{q} x(t), \quad t \in \mathbb{R}^{+}, \quad q \in Q
$$

where $\mathbb{R}^{+}$denotes non-negative real numbers. Also, we consider discrete-time switched systems with a collection of discrete-time LTI systems

$$
x[k+1]=A_{q} x[k], \quad k \in \mathbb{Z}^{+}, \quad q \in Q
$$

where $\mathbb{Z}^{+}$stands for non-negative integers. The finite set $Q$ is an index set and stands for the collection of discrete modes. The logical rule that orchestrates switching between these subsystems generates switching signals, which are usually described as classes of piecewise constant maps, $\sigma: \mathbb{R}^{+} \rightarrow Q$ (or $\sigma: \mathbb{Z}^{+} \rightarrow Q$ ). By piecewise constant, we mean that the switching signal $\sigma(t)$ (or $\sigma(k)$ ) has finite number of discontinuities on any finite interval of $\mathbb{R}^{+}$ (or $\mathbb{Z}^{+}$). This requirement is always satisfied by discretetime switched systems. However, for the continuous-time switched systems, this corresponds to no-chattering requirement. Note that the restriction that there are a finite number

\footnotetext{
The partial support of the National Science Foundation (NSF CCR0113131) is gratefully acknowledged. The valuable comments from the anonymous reviewers are highly appreciated.

H. Lin and Panos J. Antsaklis are both with the Department of Electrical Engineering, University of Notre Dame, Notre Dame, IN 46556, USA. Email: $\{$ hlin1, antsaklis.1\}@nd.edu
}

of switches in finite time does not necessarily exclude sliding-like motions. Indeed, sliding motions may be incorporated by defining each such motion and its associated equivalent dynamics [11] as an additional system to which we can switch.

Switched systems have been studied for the past fifty years to consider engineering systems that contain relays and/or hysteresis. The primary motivation for studying such switched systems comes partly from the fact that switched systems and switched multi-controller systems have numerous applications in control of mechanical systems, process control, automotive industry, power systems, aircraft and traffic control, and many other fields. In addition, there exists a large class of nonlinear systems which can be stabilized by switching control schemes, but cannot be stabilized by any continuous static state feedback control law [7]. Recent efforts in switched system research typically focus on the analysis of dynamic behaviors, such as stability [15], [17], [9], [19], controllability and observability [4], [35], [37] etc., and aim to design controllers with guaranteed stability and optimized performance [3], [15], [37].

The stability issues of switched systems, especially switched linear systems, have been of increasing interest in the recent decade, see for example the survey papers [17], [9], the recent book [19] and the references cited therein. The stability issues of switched systems include several interesting phenomena. For example, even when all the subsystems are exponentially stable, the switched systems may have divergent trajectories for certain switching signals. Another remarkable fact is that one may carefully switch between unstable subsystems to make the switched system exponentially stable. As these examples suggest, the stability of switched systems depends not only on the dynamics of each subsystem but also the properties of switching signals. Therefore, the stability study of switched systems can be roughly divided into two kinds of problems. One is the stability analysis of switched systems under given switching signals (maybe arbitrary, slow switching etc.); the other is the synthesis of stabilizing switching signals for a given collection of dynamical systems. In this current paper, we will briefly overview the stability and stabilizability of switched systems from these two aspects. In particular, Section II investigates the stability analysis problem, while the switching stabilization problem is studied in Section III.

There have been several excellent survey papers on the stability of switched systems. However, this field has seen a large amount of activities and new results since their publications. Therefore, this paper aims to briefly survey 
the recent results in this field. The authors hope that this provides some meaningful complementary resources to previous survey papers like [17], [24], [9], [37]. In particular, this paper highlights necessary and sufficient conditions for stability and stabilizability of switched linear systems, and a converse Lyapunov theorem.

\section{Stability Analysis}

\section{A. Stability Under Arbitrary Switchings}

For the stability analysis problem, the first question is whether the switched system is stable when there is no restriction (or no a priori knowledge) on the switching signals. This problem is usually called stability analysis under arbitrary switching.

For this problem, it is necessary to require that all the subsystems are asymptotically stable, since one may always stick to a certain subsystem $q$ all time, i.e., $\sigma(t)=q$ (or $\sigma(k)=q$ ) for all $t$ (or $k$ ), which is a valid 'switching signal'. However, even when all the subsystems of a switched system are exponentially stable, it is still possible to construct a divergent trajectory from any initial state for such a switched system. Therefore, in general, the above subsystems' stability assumption is not sufficient to assure stability for the switched systems under arbitrary switching signals. On the other hand, if there exists a common Lyapunov function for all the subsystems, then the stability of the switched systems is guaranteed under arbitrary switchings. This provides us a possible way to solve this problem, and a lot of attempts have been focused on common quadratic Lyapunov functions.

1) Common Quadratic Lyapunov Functions: The existence of a common quadratic Lyapunov function for all its subsystems assures the quadratic stability, a special class of exponential stability, of the switched system. However, the conditions (on the subsystems' dynamics) for the existence of a common quadratic Lyapunov function is not easy to determine. In fact, there have been various attempts to derive conditions for the existence of a common quadratic Lyapunov function, see for example [18], [19] and their references. In [32], Shorten and Narendra considered a second-order switched LTI systems with two modes; they proposed a necessary and sufficient condition, which is based on the stability of the matrix pencil formed by the pair of subsystems' state matrices, for the existence of a common quadratic Lyapunov function. This result was extended to a switched systems composed of more than two LTI subsystems in [33]. Recently, King and Shorten provided a necessary and sufficient algebraic condition for an arbitrary switching systems composed of a pair of thirdorder LTI systems [16]. They have also shown that this result could be extended to a pair of higher order LTI systems. However, for general cases of higher order and more than two modes, the necessary and sufficient condition for the existence of a common quadratic Lyapunov function for a switched LTI systems is still lacking and remains an open problem.
Alternatively, Liberzon, Hespanha and Morse proposed a Lie algebraic condition in [18] for switched LTI systems, which is based on the solvability of the Lie algebra generated by the subsystems' state matrices. Notice that the Lie algebraic conditions imply the existence of a common quadratic Lyapunov function. This is because the matrices in a solvable Lie algebra can be simultaneously put in the upper-triangular form, and that a family of linear systems with stable upper-triangular matrices possess a common quadratic Lyapunov function. The algebraic condition was also extended to switched nonlinear systems [18], [1] to obtain local stability based on Lyapunov's first method.

The problem of finding a common quadratic Lyapunov function can be solved by a collection of linear matrix inequalities (LMIs). However, the standard interior methods for LMIs may become ineffective as the number of modes increases. In [20], an interactive gradient decent algorithm was proposed, which could converge to the common quadratic Lyapunov function in finite number of steps. In addition, the authors showed that the convergence rate could be improved by introducing some randomness, where the convergence is in the sense of probability one.

It is worth pointing out that the existence of a common quadratic Lyapunov function is only sufficient for the stability of arbitrary switching systems. There are switched systems that do not have common quadratic Lyapunov functions, but are exponentially stable under arbitrary switching [19]. Consequently, most of the work based on common quadratic Lyapunov function mentioned above only gave sufficient stability test criteria for arbitrary switching linear systems except for some special cases. Next, we will discuss these special cases where the quadratic stability is equivalent to asymptotic stability and the subsystems' stability assures the existence of a quadratic Lyapunov function and the stability of the arbitrary switching system.

2) Some Special Cases: One of such special cases is the case of a switched LTI system whose subsystems' state matrices are pairwise commutative [27], [40], i.e., $A_{i} A_{j}=$ $A_{j} A_{i}$ for all $i, j \in Q$. Because of the commutativity, it is easy to derive that

$$
A_{i}^{k_{1}} A_{j}^{k_{2}}=A_{j}^{k_{2}} A_{i}^{k_{1}},
$$

for any nonnegative integer $k_{1}$ and $k_{2}$, and

$$
e^{A_{i} t_{1}} e^{A_{j} t_{2}}=e^{A_{j} t_{2}} e^{A_{i} t_{1}},
$$

for any nonnegative real number $t_{1}$ and $t_{2}$. By direct computation, it is straightforward to verify that the switched systems is stable if and only if all its subsystems are stable. In addition, a common quadratic Lyapunov function can be determined by solving a collection of chained Lyapunov equations.

Another interesting case is when all the subsystems' state matrices $A_{q}$ are symmetric [40], i.e., $A_{q}=A_{q}^{T}$, where $A_{q}^{T}$ stands for the transpose of matrix $A_{q}$. In fact, the square of the state's Euclidian norm serves as a common quadratic Lyapunov function for both discrete- and continuous-time 
switched symmetric systems. To verify this, note that the identity matrix satisfies all the subsystems Lyapunov equations.

A less well known case is a switched normal system. A switched normal system is a switched LTI system whose subsystems are normal, i.e., $A_{q} A_{q}^{T}=A_{q}^{T} A_{q}$ for every mode $q$. It can be shown that the square of the state's Euclidian norm also serves as a common quadratic Lyapunov function for switched normal systems. In summary, we have the following result.

Proposition 1: The necessary and sufficient condition for a switched commutative (symmetric, or normal) LTI system being asymptotically stable is that all its subsystems are asymptotically stable.

3) A Necessary and Sufficient Condition: So far, we have only obtained sufficient test criteria for stability of arbitrary switching systems, except in certain special cases. In the sequel, we will provide a necessary and sufficient condition for the asymptotic stability of switched linear systems with arbitrary switching signals [22]. This is a relatively new result which provide a solution for this long standing problem. It shows that the asymptotic stability for switched linear systems with arbitrary switching is equivalent to the robust asymptotic stability for polytopic uncertain linear time-variant systems.

Let us first introduce a technical lemma [2] for the robust stability of linear time variant systems with polytopic uncertainty

$$
x[k+1]=A(k) x[k]
$$

where $A(k) \in \mathcal{A} \hat{=} \operatorname{Conv}\left\{A_{1}, A_{2}, \cdots, A_{\mathbf{N}}\right\}$. Here, Conv $\{\cdot\}$ stands for convex combination.

Lemma 1: The linear time-variant system (3) is globally asymptotically stable if and only if there exists a finite integer $\mathbf{n}$ such that $\left\|A_{i_{1}} A_{i_{2}} \cdots A_{i_{\mathbf{n}}}\right\|<1$, for all $\mathbf{n}$-tuple $A_{i_{j}} \in \operatorname{vert}\{\mathcal{A}\}=\left\{A_{1}, A_{2}, \cdots, A_{\mathbf{N}}\right\}$, where $j=1, \cdots, \mathbf{n}$.

Here the norm $\|\cdot\|$ stands for either 1 norm or $\infty$ norm of a matrix. The asymptotic stability of the switched system can be expressed as the following proposition.

Proposition 2: A switched linear system $x[k+1]=$ $A_{\sigma(k)} x[k]$, where $A_{\sigma(k)} \in\left\{A_{1}, A_{2}, \cdots, A_{\mathbf{N}}\right\}$, is globally asymptotically stable under arbitrary switchings if and only if there exists a finite integer $\mathbf{n}$ such that

$$
\left\|A_{i_{1}} A_{i_{2}} \cdots A_{i_{\mathbf{n}}}\right\|<1,
$$

for all $\mathbf{n}$-tuple $A_{i_{j}} \in\left\{A_{1}, A_{2}, \cdots, A_{\mathbf{N}}\right\}$, where $j=$ $1, \cdots, \mathbf{n}$.

The sufficiency of the above condition is implied by Lemma 1, and the necessity can be easily shown by contradiction. Notice that this condition coincides with the necessary and sufficient condition for the robust asymptotic stability for polytopic uncertain linear time-variant systems (3). Therefore, we derive the equivalence relationship between these two problems. It is quite interesting that the study of robust stability of a polytopic uncertain linear time-variant system, which has infinite number of possible dynamics (modes), is equivalent to only considering a finite number of its vertex dynamics as an arbitrary switching system. In fact, it is not a surprising result since this fact has already been implied by the finite vertex stability criteria for robust stability in the literature, e.g., [26], [5]. By explicitly exploring this equivalence relationship, we may obtain some "new" stability criteria for switched linear systems using the existing robust stability results.

Although we only discuss here the discrete-time case, this result is also true in the continuous-time case. This fact bridges two distinct research fields. Therefore, existing results in the robust stability area, which has been extensively studied for over two decades, can be directly introduced to study the arbitrarily switching systems and vice versa. In the next subsection, we will present a converse Lyapunov function for arbitrary switching systems, which is known in the literature of absolute stability for linear time varying systems.

4) Converse Lyapunov Theorem: In [8], a converse Lyapunov theorem was derived for the globally uniformly asymptotically stable and locally uniformly exponentially stable continuous-time switched systems with arbitrary switching signals. It was shown that such arbitrary switching system admits a common Lyapunov function. The converse Lyapunov theorem was extended in [23] to switched nonlinear systems that are globally uniformly asymptotically stable with respect to a compact forward invariant set. These converse Lyapunov theorems justify the common Lyapunov method being pursued. However, they also suggest that the common Lyapunov function may not necessarily be quadratic, although most of the available results pertain to the existence of common quadratic Lyapunov functions. Therefore, the study of non-quadratic Lyapunov function, especially polyhedral Lyapunov function, has been attracting more and more attention.

Below we present a converse Lyapunov theorem, where the Lyapunov function is non-quadratic, in particular polyhedral Lyapunov function (also known as piecewise linear Lyapunov function). This is a simple implementation of the equivalence between the asymptotic stability of arbitrary switching linear systems and the robust stability of polytopic uncertain linear time varying systems.

Proposition 3: If a switched LTI system system is asymptotically stable under arbitrary switching signals, then there exists a polyhedral Lyapunov function, which is monotonically decreasing along the switched system's trajectories.

This converse Lyapunov theorem holds for both discretetime and continuous-time cases. In addition, for switched linear systems under arbitrary switching signals, the following stability concepts are equivalent: asymptotic stability, global asymptotic stability, and (global) exponential stability. 


\section{B. Stability Under Restricted Switchings}

Switched systems may fail to preserve stability under arbitrary switchings. By studying the example in [17], [9] where divergent trajectories are generated through switching between two stable systems, one may notice that the unboundedness is caused by the failure to absorb the energy increase caused by the switching. In addition, when there is an unstable subsystem (e.g., controller failure or sensor fault), it may cost stability if one either stays too long at or switches too frequently to the unstable subsystem. Therefore, a natural question is what if we restrict the switching signal to some constrained subclass of switchings. Intuitively, if one stays at stable subsystems long enough and switches less frequently, i.e., slow switching, one may trade off the energy increase caused by switching or unstable modes, and it is possible to attain stability. These ideas are proved to be reasonable and are captured by concepts like dwell time and average dwell time switching in the literature, see for example [12], [13].

In this subsection, it is assumed that the switching signals are restricted, and our problem is to study the stability of the switched systems under these restricted switching signals. With this problem being solved, one could provide an answer to the question regarding what restrictions should be put on the switching signals in order to guarantee the stability of switched systems. The restrictions on switching signals may be either time domain restrictions (e.g., dwell-time, average dwell-time switchings) or state space restrictions (e.g. abstractions from partitions of the state space). Notice that the distinction between time-controlled switching signals (trajectory independent) and trajectory dependent switching signals is significant. In [13], Hespanha showed that when the class of switching signals is timecontrolled, i.e., trajectory independent, uniform asymptotic stability of switched linear systems is equivalent to exponential stability. However, this equivalence does not hold for trajectory dependent switching signals. A counter example is given in [13].

The stability analysis with constrained switchings has been usually pursued in the framework of multiple Lyapunov functions (MLF). The basic idea is using multiple Lyapunov or Lyapunov-like functions, which may correspond to each single subsystem or certain region in the state space, concatenated together to produce a non-traditional Lyapunov function. The non-traditionality is in the sense that the MLF may not be monotonically decreasing along the state trajectories, may have discontinuities and be piecewise differentiable. The reason for considering nontraditional Lyapunov functions is that traditional Lyapunov function may not exist for switched systems with restricted switching signals. In other words, the converse Lyapunov theorems discussed before for switched systems under arbitrary switching, i.e., without restriction on switching signals, may fail to hold for switched systems with restricted switching.
MLF is proved to be a powerful tool for studying the stability of switched systems, see for example [6]. The basic idea of MLF method can be described as follows. If we assume that all subsystems are stable, then each subsystem can be associated with a Lyapunov function. It is known that the Lyapunov function's value would decrease when the corresponding subsystem is active. If we restrict the switching signals in such a way that, at every time when we enter (switch into) a certain subsystem, its corresponding Lyapunov function value is smaller than its value at the previous entering time, then the switched system is asymptotically stable. In other words, for each subsystem the corresponding Lyapunov function value at every entering instant form a monotonically decreasing sequence.

If not all the subsystems are asymptotically stable, then there may not exist a Lyapunov function in the traditional sense for these subsystems. For such cases, one may construct a Lyapunov-like function, which only requires nonpositive Lie-derivative in a certain region of the state space instead of negative globally. By patching these Lyapunovlike functions together, similar MLF results can be derived for the switched systems. Furthermore, the Lyapunov-like function may increase its value during a time interval, only if the increment is bounded by certain kind of continuous functions [39]. There are various extensions of MLF results; interested readers may refer to survey papers [9], [17] and their references.

Implicitly, the above MLF results provide methodologies for switching between vector fields to achieve a stable trajectory. In the next section, we will focus on this topic.

\section{Switching Stabilization}

In the previous section, we discussed stability properties of a switched systems under given switching signals, which may be restricted or arbitrary (without restriction). The problem studied was under what conditions, either on the subsystems' dynamics or on the switching signals, the switched system is stable, which is basically a stability analysis problem. Another basic problem for switched systems is the synthesis of stabilizing switching laws for a given collection of dynamical systems, which is called the switching stabilization problem.

\section{A. Quadratic Switching Stabilization}

In the switching stabilization literature, most of the work has focused on quadratic stabilization for certain classes of systems. For example, a quadratic stabilization switching law between two LTI systems was considered in [31], in which it was shown that the existence of a stable convex combination of the two subsystem matrices implies the existence of a state-dependent switching rule that stabilizes the switched system along with a quadratic Lyapunov function. A generalization to more than two LTI subsystems was suggested in [28] by using a "min-projection strategy". In [10], it was shown that the stable convex combination 
condition is also necessary for the quadratic stabilizability of two mode switched LTI system. However, it is only sufficient for switched LTI systems with more than two modes.

Unfortunately, finding the stable convex combination of state matrices is an NP-hard problem [34]. Moreover, there is a large class of systems for which no stable convex combination state matrix exists, yet there exists a stabilizing switching law. A necessary and sufficient condition for quadratic stabilizability of switched controller systems was derived in [34]. There are extensions of [31] to outputdependent switching and discrete-time case [17], [41]. For robust stabilization, a quadratic stabilizing switching law was designed for polytopic uncertain switched linear systems based on LMI techniques in [41]. All of these methods guarantee stability by using a common quadratic Lyapunov function, which is conservative in the sense that there are switched systems that can be asymptotically (or exponentially) stabilized without using a common quadratic Lyapunov function.

\section{B. Based on Multiple Lyapunov Functions}

There have been some results in the literature that propose constructive synthesis methods using multiple Lyapunov functions. The first stabilizing switching law design based on multiple Lyapunov functions was proposed in [30], where piecewise quadratic Lyapunov functions was employed for two mode switched LTI systems. An LMI based method was proposed in [25] for the stabilizing statefeedback control design of discrete-time piecewise affine systems. Exponential stabilization for switched LTI systems was considered in [29] also based on piecewise quadratic Lyapunov functions, and the synthesis problem was formulated as a bilinear matrix inequality (BMI) problem. In [14], a probabilistic algorithm was proposed for the synthesis of an asymptotically stabilizing switching law for switched LTI systems along with a piecewise quadratic Lyapunov function. Notice that these stabilizability conditions, which may be expressed as the feasibility of certain LMIs or BMIs, in the existing literature are basically sufficient only, except for certain cases of quadratic stabilization.

\section{Switching Stabilizability}

One of the most elusive problems in the switched systems literature has been the switching stabilizability problem, that is under what condition it is possible to stabilize a switched system by properly designing switching control laws.

In [36], Sun proved the following necessary condition for switching stabilizability.

Proposition 4: If there exist an asymptotically stabilizing switching signal among a finite number of LTI systems

$$
\dot{x}(t)=A_{i} x(t),
$$

where $i=1,2, \cdots, N$, then there exists a subsystem, say $A_{k}$, such that at least one of the eigenvalues of $A_{k}+A_{k}^{T}$ is a negative real number.
This condition can be easily checked, but it is necessary only. A necessary and sufficient condition for asymptotic stabilizability of second-order switched LTI systems was derived in [38] by detailed vector field analysis. However, it was not apparent how to extend the method to either higher dimensions or to the parametric uncertainty case.

Recently, Lin and Antsaklis proposed a necessary and sufficient condition for switching stabilizability for continuous-time switched linear systems in [21].

For each unstable subsystem,

$$
\dot{x}(t)=A_{q} x(t)
$$

it is assumed that there exists a full row rank matrix $L_{q} \in$ $\mathbb{R}^{m_{q} \times n}$, where $m_{q}<n$, such that the auxiliary system for the $q$-th subsystem

$$
\dot{\xi}(t)=L_{q} A_{q} R_{q} \xi(t), \quad t \in \mathbb{R}^{+}
$$

is asymptotically stable. Here $R_{q} \in \mathbb{R}^{n \times m_{q}}$ is the right inverse of $L_{q}$.

Intuitively, the above assumption can be interpreted as considering a linear combination of the states of the original system (4) that evolves in an asymptotically stable manner. The auxiliary system evolves in the lower dimensional subspace to which the original system can be projected for stability. In fact, it can be shown that there always exist $L$ and $R$ satisfying the above assumption, except for the case when all the eigenvalues of $A$ are the same positive real number $\lambda>0$ and the geometric multiplicity of the eigenvalue $\lambda$ equals to $n$. The proof of this claim explores the structure of the Jordan canonical form of $A$ and uses straight-forward computations.

For the case when there does not exist an $L$ to satisfy the above assumption for a particular subsystem, we simply set $L$ as the null row vector, which implies that the corresponding subsystem makes no contribution to the stabilization of the switched system. To justify this, note that in this case the matrix $A$ is similar to the matrix $\lambda I$ for some positive real number $\lambda>0$. Here $I$ stands for the identity matrix. If we look at the phase plane of the LTI system, $\dot{x}(t)=\lambda I x(t)$, all the field vectors point to infinity along the radial directions. Intuitively speaking, the dynamics are explosive and do nothing but drag all the states to infinity, which we would like to avoid.

The basic idea is that a polyhedral Lyapunov-like function can be constructed for each subsystem by transforming the polyhedral Lyapunov function from its corresponding auxiliary system. Notice that every auxiliary system is asymptotically stable, so such polyhedral Lyapunov functions always exist [26]. An important observation is that for each subsystem the polyhedral Lyapunov-like function is decreasing for all the state $x$ in the range space of $R_{q}$.

If for all the subsystems, the matrix

$$
\left[\begin{array}{cccc}
L_{1}^{T} & L_{2}^{T} & \ldots & L_{N}^{T}
\end{array}\right]^{T} \in \mathbb{R}^{\sum_{q} m_{q} \times n},
$$

has full row rank and the union of the range space of $R_{q} \mathrm{~s}$ ' is the whole state space, then an asymptotically stabilizing 
switching law can be constructed. This shows the sufficiency of the above condition. In addition, it is shown that the condition is also necessary for switching stabilizability. The necessity proof is based on the lemma that a switched linear system can be asymptotically stabilized if there exists a conic partition based switching law. Interested readers may find technique details in [21].

\section{CONCluding Remarks}

In this paper, we gave a brief overview of the most recent developments in the field of stability and stabilizability of switched linear systems. Some new results were reported in this paper. This short survey is far from a complete review of stability and stabilizability of switched systems. Unavoidably, there are papers that are not mentioned here either due to space limit or due to the authors' knowledge limit. We apologize for these omissions.

\section{REFERENCES}

[1] A. A. Agrachev and D. Liberzon, "Lie-algebraic stability criteria for switched systems," SIAM J. Control Optim., vol. 40, pp. 253-270, 2001.

[2] P. Bauer, K. Premaratne, and J. Duran, "A necessary and sufficient condition for robust asymptotic stability of time-variant discrete systems," IEEE Trans. AC, vol. 38, no. 9, pp. 1427-1430, 1993.

[3] A. Bemporad and M. Morari, "Control of systems integrating logic, dynamics, and constraints," Automatica, vol. 35, no. 3, pp. 407-427, 1999.

[4] A. Bemporad, G. Ferrari-Trecate, and M. Morari, "Observability and controllability of piecewise affine and hybrid systems," IEEE Trans. $A C$, vol. 45, no. 10, pp. 1864-1876, 2000.

[5] A. Bhaya and F. Mota, "Equivalence of stability concepts for discrete time-varying systems," Int. J. Robust and Nonlin. Contr., vol. 4, pp. 725-740, 1994.

[6] M. S. Branicky, "Multiple Lyapunov functions and other analysis tools for switched and hybrid systems," IEEE Trans. AC, vol. 43, no. 4, pp. 475-482, 1998.

[7] R. W. Brockett, "Asymptotic stability and feedback stabilization," in Differential Geometric Control Theory, R. W. Brockett, R. S. Millman, and H. J. Sussmann, Eds. Boston, MA: Birkhuser, pp. 181-191, 1983.

[8] W. Dayawansa and C. F. Martin, "A converse Lyapunov theorem for a class of dynamical systems which undergo switching," IEEE Trans. AC, vol. 44, no. 4, pp. 751-760, 1999.

[9] R. A. Decarlo, M. S. Branicky, S. Pettersson, and B. Lennartson, "Perspectives and results on the stability and stabilizability of hybrid systems," Proceedings of the IEEE, Special issue on Hybrid Systems, P. J. Antsaklis Ed., vol. 88, no. 7, pp. 1069-1082, 2000.

[10] E. Feron, "Quadratic stabilizability of switched systems via state and output feedback," technique report, CICS-P-468, MIT, 1996.

[11] A. F. Filippov, Differential Equations with Discontinuous Right Hand Sides, Norwell, MA: Kluwer Academic, 1988.

[12] J. P. Hespanha and A. S. Morse, "Stability of switched systems with average dwell-time," in Proc. 38th IEEE Conf. Decision Control, pp. 2655-2660, 1999.

[13] J. P. Hespanha, "Uniform stability of switched linear systems: Extensions of LaSalle's invariance principle," IEEE Trans. AC, vol. 49, no. 4, pp. 470-482, 2004

[14] H. Ishii, T. Basar, and R. Tempo, "Synthesis of switching rules for switched linear systems through randomized algorithms," in Proc. 42nd IEEE Conf. Decision Control, pp. 4788-4793, 2003.

[15] M. Johansson, Piecewise Linear Control Systems, Ph.D. Thesis, Lund Institute of Technology, Sweden, 1999.

[16] C. King and R. Shorten, "A singularity test for the existence of common quadratic Lyapunov functions for pairs of stable LTI systems," in Proc. 2004 American Contr. Conf., pp. 3881-3884, 2004.
[17] D. Liberzon and A. S. Morse, "Basic problems in stability and design of switched systems," IEEE Contr. Syst. Magazine, vol. 19, no. 5, pp. 59-70, 1999.

[18] D. Liberzon, J. P. Hespanha and A. S. Morse, "Stability of switched linear systems: A Lie-algebraic condition," Syst. Contr. Lett., vol. 37, no. 3, pp. 117-122, 1999.

[19] D. Liberzon, Switching in Systems and Control, Birkhauser, Boston, 2003.

[20] D. Liberzon and R. Tempo, "Common Lyapunov functions and gradient algorithms," IEEE Trans. AC, vol. 49, no. 6, pp. 990-994, 2004.

[21] H. Lin and P. J. Antsaklis, "A necessary and sufficient condition for robust asymptotic stabilizability of continuous-time uncertain switched linear systems," in Proc. 43rd Conf. Decision Control, pp. 3690-3695, 2004.

[22] H. Lin and P. J. Antsaklis, "Persistent disturbance attenuation properties for networked control systems," in Proc. 43rd Conf. Decision Control, pp. 953-958, 2004.

[23] J. L. Mancilla-Aguilar and R. A. García, "A converse Lyapunov theorem for nonlinear switched systems," Systems \& Control Letters, vol. 41, no. 1 , pp. 67-71, 2000.

[24] A. N. Michel, "Recent trends in the stability analysis of hybrid dynamical systems," IEEE Trans. Circuits Syst. I, vol. 46, no. 1, pp. 120-134, 1999.

[25] D. Mignone, G. Ferrari-Trecate, and M. Morari, "Stability and stabilization of piecewise affine and hybrid systems: An LMI approach," in Proc. 39th Conf. Decision Control, pp. 504-509, 2000.

[26] A. Molchanov and E. Pyatnitskiy, "Criteria of asymptotic stability of differential and difference inclusions encountered in control theory," Syst. Contr. Lett., vol. 13, pp. 59-64, 1989.

[27] K. S. Narendra and J. Balakrishnan, "A common Lyapunov function for stable LTI systems with commuting A-matrices," IEEE Trans. $A C$, vol. 39, no. 12, pp. 2469-2471, 1994.

[28] S. Pettersson and B. Lennartson, "Stabilization of hybrid systems using a min-projection strategy," in Proc. 2001 American Contr. Conf., pp. 223-228, 2001.

[29] S. Pettersson, "Synthesis of switched linear systems," in Proc. 42nd IEEE Conf. Decision Control, pp. 5283-5288, 2003.

[30] M. A. Wicks and R. A. DeCarlo, "Solution of coupled Lyapunov equations for the stabilization of multimodal linear systems," in Proc. 1997 American Contr. Conf., pp. 1709-1713, 1997.

[31] M. A. Wicks, P. Peleties, and R. A. DeCarlo, "Switched controller design for the quadratic stabilization of a pair of unstable linear systems," European J. Control, vol. 4, pp. 140-147, 1998.

[32] R. Shorten and K. Narendra, "Necessary and sufficient conditions for the existence of a common quadratic Lyapunov function for two stable second order linear time-invariant systems," in Proc. 1999 American Contr. Conf., pp. 1410 - 1414, 1999.

[33] R. Shorten and K. Narendra, "Necessary and sufficient conditions for the existence of a CQLF for a finite number of stable LTI systems," International Journal of Adaptive Control and Signal Processing, vol. 16, no. 10, pp. 709-728, 2002.

[34] E. Skafidas, R. J. Evans, A. V. Savkin, and I. R. Petersen, "Stability results for switched controller systems," Automatica, vol. 35, no. 4, pp. 553-564, 1999.

[35] Z. Sun, S. S. Ge, and T. H. Lee, "Controllability and reachability criteria for switched linear systems," Automatica, vol. 38, no. 5, pp. 775-786, 2002.

[36] Z. Sun, "Stabilizability and insensitivity of switched linear systems," IEEE Trans. AC, vol. 49, no. 7, pp. 1133-1137, 2004.

[37] Z. Sun and S. S. Ge, "Analysis and synthesis of switched linear control systems," Automatica, vol. 41, no. 2, pp. 181-195, 2005.

[38] X. Xu and P. J. Antsaklis, "Stabilization of second-order LTI switched systems," Inter. J. Contr., vol. 73, no. 14, pp. 1261-1279, 2000.

[39] H. Ye, A. N. Michel, and L. Hou, "Stability theory for hybrid dynamical systems," IEEE Trans. AC, vol. 43, no. 4, pp. 461-474, 1998.

[40] G. Zhai, B. Hu, K. Yasuda, and A. N. Michel, "Qualitative analysis of discrete-time switched systems," in Proc. 2002 American Contr. Conf., vol. 3, pp. 1880-1885, 2002.

[41] G. Zhai, H. Lin, and P. J. Antsaklis, "Quadratic stabilizability of switched linear systems with polytopic uncertainties," Inter. J. Contr., vol. 76, no. 7, pp. 747-753, 2003. 